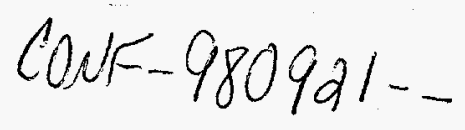

\title{
MATERIALS COMPATIBILITY STUDIES FOR THE SPALLATION NEUTRON SOURCE
}

\author{
J. R. DiStefano, S. J. Pawel, and E. T. Manneschmidt \\ Oak Ridge National Laboratory \\ P.O. Box 2008 \\ Oak Ridge, TN 37831-6157 \\ (423) $574-4452$
}

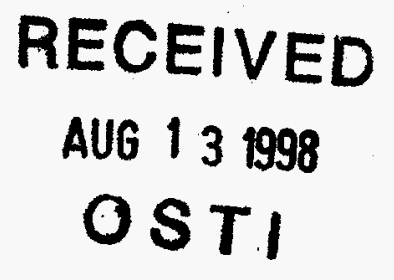

\section{INTRODUCTION}

The Spallation Neutron Source (SNS) is a high power facility for producing neutrons that utilizes flowing liquid mercury inside an austenitic stainless steel container as the target for a $1.0 \mathrm{GeV}$ proton beam. The energy deposited in the target is transported by two separate mercury flow streams: one to transport heat in the interior target region and one to cool the stainless steel container. Threedimensional computational fluid dynamics simulations have been performed to predict temperature, velocity, and pressure distributions in the target. Results have generally shown that the power deposited in the bulk mercury can be effectively transported with reasonable flow rates and the bulk mercury temperature should not exceed $160^{\circ} \mathrm{C}$. Assuming good thermal contact, the maximum stainless steel wall temperature should be $130^{\circ} \mathrm{C}$. Type $316 \mathrm{SS}$ has been selected as the container material for the mercury and consequences of exposure of $316 \mathrm{SS}$ to radiation, thermal shock, thermal stress, cavitation and hot, flowing mercury are all being addressed by $R \& D$ programs. In addition, corrosion studies also include evaluation of Inconel 718 because it has been successfully used in previous spallation neutron systems as a window material.

Two types of compatibility issues relative to $316 \mathrm{SS} /$ mercury and Inconel 718/mercury are being examined: (1) liquid metal embrittlement (LME) and (2) temperature gradient mass transfer. Although the phenomenon of LME has been known for over 100 years, it is still not well understood. ${ }^{\prime}$ For LME to occur, a tensile-stressed metal must be wetted by the liquid. Without wetting LME is unlikely, but extensive chemical corrosion by the liquid usually means no LME. In fact, LME is usually most severe near the melting temperature of the liquid and decreases as the temperature is increased. Mercury is known to embrittle several metals, e.g., aluminum, nickel, zinc, and titanium. ${ }^{2}$ Hayden and Floreen $^{3}$ also found that Fe-Ni was embrittled by $\mathrm{Hg}-0.1 \%$
In, and Krupowicz $z^{4}$ indicated that types 304 and 304L SS were somewhat embrittled by $\mathrm{Hg}$ in slow strain rate tests at room temperature, but types 316 and 316L SS were not.

Temperature gradient mass transfer is a form of corrosion that results from dissolution by the liquid in the higher temperature regions of the system and subsequent deposition of solute in colder regions because of supersaturation. Nickel in 316 SS or Inconel 718 has the highest relative solubility of the elements in these alloys and should be the most susceptible to mass transfer; however, if solid state diffusion limits the supply of nickel at the surface, preferential corrosion of nickel would be suppressed.

\section{PRELIMINARY EVALUATION OF WETTING BY MERCURY}

Some form of wetting by mercury is required for cooling of the target container. Therefore, prior to conducting LME or mass transfer studies, some qualitative wetting experiments were conducted. In these studies, we found that both stainless steels and nickel-based alloys were resistant to wetting by mercury, particularly at or near room temperature. For example, U-bend samples of type $304 \mathrm{~L}$ SS were ultrasonically cleaned and then treated as follows:

1. no additional treatment,

2. gold plated $(10 \mu \mathrm{m})$,

3. acid cleaned with acid subsequently displaced by mercury, and

4. specimen abraided under mercury.

The samples were then exposed to mercury in air for $72 \mathrm{~h}$ at $25,150,225$, and $275^{\circ} \mathrm{C}$. There was no discernible wetting until $225^{\circ} \mathrm{C}$ (very slight) and $275^{\circ} \mathrm{C}$. Gallium is a highly active liquid metal that has some solubility in mercury even at room temperature. When small amounts
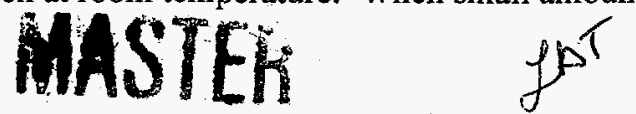